\title{
EXTENDING EVERYTHING WITH NOTHING
}

\section{Filippo Costantini}

[This is Post-print Manuscript (Author Accepted Version) of a paper published in Philosophia 48, 1413-1436 (2020), Springer Verlag; DOI: 10.1007/s11406-019-00144-x; please quote from the final version: https://link.springer.com/article/10.1007\%2Fs11406019-00144-x\#citeas]

Abstract: In this paper we offer a new solution to the old paradox of nothingness. This new solution develops in two steps. The first step consists in showing how to resolve the contradiction generated by the notion of nothingness by claiming that the contradiction shows the indefinite extensibility of the concept of object. The second step consists in showing that, having accepted the idea of indefinite extensibility, we can have absolute generality without the emergence of the contradiction connected to the absolute notion of nothingness. The idea of indefinite extensibility allows us to have our cake (absolute generality) and to eat it too (avoid commitment to a contradictory notion of nothingness).

Key-words: Everything, Nothing (paradox of), Indefinite Extensibility, Absolute Generality

\section{§1. Introduction}

In this paper we offer a new solution to the old paradox of nothingness. Historically ${ }^{1}$ the problem has been raised in the following form: it is not possible to refer to nothingness, since it is not (it does not exist). But in order to claim that we cannot refer to nothingness, we must speak of it, i.e. we must refer to it. The same claim that we cannot refer to nothingness actually entails referring to it. So, nothingness seems to be a contradictory notion.

More recently, the notion of nothingness has received quite a lot of attention in analytic metaphysics ${ }^{2}$. There are a number of reasons that explain such an interest. Some philosophers, such as Priest (2014), have argued that the standard Carnapian view according to which the only meaningful occurrence of 'nothing' is the quantificational one fails, since there are occurrences of 'nothing' as a noun phrase which cannot be

\footnotetext{
1 The first work where this paradox has emerged is Parmenides's poem On Nature (see Coxon 2009). After Parmenides the problem has been constantly studied in the history of philosophy: here I limit myself to refer to Plato's Sophiest (see Cooper 1997) and to the opening of Hegel's Science of Logic.

2 See in particular Voltolini 2015, Oliver and Smiley 2013, Priest 2014, Casati and Fujikawa 2017, and Simionato 2017.
} 
paraphrased away by means of a quantifier without altering the meaning of the sentence ${ }^{3}$. Others (e.g. Oliver and Smiley 2013) have argued that 'nothing' as an empty term has a perfectly good use in English, and thus we - as logicians - should be interested in studying its logical behavior. Finally, a logical study of 'nothingness' as a singular term can be useful to interpret philosophers that spoke of nothingness, such as Heidegger (see Voltolini 2015, Casati and Fujikawa 2015) or Hegel (see Simionato 2015, 2018). In this paper, I shall assume the legitimacy of 'nothingness' as a proper singular term, which implies that the paradox of nothingness is a truly philosophical one deserving of a solution.

A full discussion and critique of all such accounts exceed the limits of the present work. I shall therefore limit myself to some sporadic comments on some of those accounts while developing an alternative account to solve the paradox of nothingness. This alternative account exploits some ideas taken from the debate on the foundation of mathematics. In particular, the proposal analyses the prospects for the problem of nothingness in a setting where indefinitely extensible concepts are accepted. To be more precise, the contradiction that the notion of nothingness implies will be exploited to argue that the concept of object is indefinitely extensible. In this way, the paper gives a new argument for (a certain conception of) indefinite extensibility. The adjective 'new' indicates that the argument does not rely on fundamental mathematical concepts that the friends of indefinite extensibility consider as paradigmatic cases of such a phenomenon, like the concept of set, ordinal, and cardinal number.

For explanatory reasons, I prefer to begin with the notion of indefinite extensibility. This is a complex notion, and $\S 2$ is devoted to presenting a rough idea of it. I limit myself to the essential considerations needed to appreciate the solution proposed here, and so the reader must not expect anything approaching a full account of such a phenomenon. I have decided to start with indefinite extensibility not because this notion is presupposed by the present solution (in fact, it is not), but merely out of simplicity and clarity. When I shall introduce my solution of the paradox of nothingness, the reader will have the essential notions connected to indefinite extensibility that will allow him to properly understand the solution. $\$ 3$ introduces Priest's account of nothingness (with some minor modifications) which I take as a basis to introduce my solution. My own solution is made up of two steps. The first step (§4) consists

3 I will give an example in footnote 14 and another in footnote 15. 
in showing how to resolve the contradiction provoked by the notion of nothingness by claiming that the contradiction shows the indefinite extensibility of the concept of object. The account that emerges is a dynamic abstractionist account ${ }^{4}$ for the notion of nothingness: such a notion is introduced by an abstraction principle with the effect that this introduction makes the domain of discourse expand. The second step (§5) shows how we can preserve absolute generality without falling back into the contradiction. In this way, I argue, the problem of nothingness is dissolved, since once indefinite extensibility has been accepted, no absolute notion of nothingness arises. $\$ 6$ discusses two objections, while $\S 7$ sketches some reasons why we should be able to live without an absolute notion of nothingness. §8 concludes.

\section{§2. Indefinite extensibility}

Dummett (1993: 441) characterizes an indefinitely extensible concept as follows:

An indefinitely extensible concept is one such that, if we can form a definite conception of a totality all of whose members fall under the concept, we can, by reference to that totality, characterize a larger totality all of whose members fall under it.

In other words, an indefinitely extensible concept $C$ is a concept associated with a principle of extension which takes a definite totality of objects $c$ falling under $\mathrm{C}$ and produces a new object that is a $\mathrm{C}$ but it is not one of the $c$ in the definite totality.

The problem with such a characterization consists in making sense of the meaning of 'definite conception of a totality'. When do we have a definite conception of a totality? In short: when can we speak of a definite totality? Of course, a totality is definite if it is not indefinitely extensible ${ }^{5}$, but this explanation is of little help, since it is obviously circular. A natural suggestion consists in identifying a definite totality either with a set or with a plurality of objects 6 . The suggestion is natural because both sets and pluralities (at least as I shall

\footnotetext{
4 The best presentation and defense of dynamic abstraction is Linnebo 2018.

5 It is tempting to speak of an indefinitely extensible totality, but this is just loose talk to say that a concept corresponds to an indefinitely extensible sequence of definite totalities. In particular, if a totality is considered to be a set or a plurality, then - properly speaking - no totality is extensible, because adding an element to a set or to a plurality results in a new set and a new plurality. What is properly indefinitely extensible is a concept, and as a consequence, the sequence of its extensions.

${ }^{6}$ Here the word 'plurality' must be taken as in plural logic, i.e. as a plural term denoting several things at once. A plurality is not a further object with regard to its elements; rather, it is simply these objects. The same term 'plurality' is just loose talk to be substituted with plural terms. Instead of saying 'a plurality of dogs' one should rather say 'the dogs'. For an overview of plural logic, see Linnebo 2017; for a more in-depth study, see Oliver
} 
consider them here) have an extensional nature. In this way a totality would be definite if its extension is fully determined from an extensional point of view. The identity principle for sets is the well-known axiom of extensionality:

$$
\text { (Est-S) } \forall x \forall y(x=y \leftrightarrow \forall u(u \in x \leftrightarrow u \in y)),
$$

where $x, y$ are first-order variables for sets, and $\in$ is the membership predicate. Given extensionality, a (particular) set $x$ is never extensible: if we enlarge a set $a$ by adding even just one more element, by (Est-S) we obtain a new set $a^{\prime}$ such that $a \neq a^{\prime}$.

The identity-principle for pluralities is a plural version of extensionality:

$$
\text { (Ext-P) } \forall x x \forall y y(x x \equiv y y \leftrightarrow \forall u(u \prec x x \leftrightarrow u \prec y y)) \text {, }
$$

where $x x, y y$ are plural first-order variables for pluralities, $u \prec x x$ is to be read as ' $u$ is one of the $x X^{\prime}$, and $\equiv$ denotes a 'congruence' relation between pluralities analogous to identity between objects (i.e. it is an equivalence relation satisfying Leibniz's law). By adding a single object to a plurality $a a$, by Ext-P we obtain a plurality $a a^{\prime}$ such that $a a \neq a a^{\prime}$.

By interpreting a definite totality with either a set or a plurality, we obtain two different conceptions of indefinite extensibility (for short: IE):

IE-1: A concept is indefinitely extensible if, given a set of objects falling under it, by reference to this set we find new objects that fall under the concept, but are not members of the original set.

(IE-2) A concept is indefinitely extensible if, given a plurality of objects (some objects) falling under it, by reference to this plurality (to these objects) we find new objects that fall under the concept, but are not members of the original plurality.

Since sets and pluralities have an extensional nature, it should be clear that an immediate upshot of the identification of definite totalities with them is that (indefinitely extensible) concepts turn out to be intensional entities that cannot be identified either with the set of all their instances (in the case of IE-1) or with the plurality of all their instances (in the case of IE-2), simply because these sets or pluralities do not exist.

The two conceptions of indefinite extensibility here introduced are not equivalent, as we shall make clear below, when we shall also explain why only IE-2 can be useful in dealing

and Smiley 2013. Linnebo (2018, pp. 57-58) also argues in favor of the identification of definite totalities with pluralities of objects. 
with the problem of nothingness ${ }^{7}$. However, before doing so, we must briefly discuss a related matter.

IE-1 generalizes over arbitrary sets of objects, while IE-2 generalizes over arbitrary pluralities. But how are we to interpret generality in the presence of an indefinitely extensible concept? How can we claim that for any set/plurality of objects falling under an indefinitely extensible concept $\mathrm{P}$, we are able to find a new object falling under $\mathrm{P}$ ? Let us consider the following two sentences, the first related to IE-1, the second to IE-2:

A. For any set of objects falling under an indefinitely extensible concept $P$, there is a more comprehensive set of objects falling under $\mathrm{P}$;

B. For any plurality of objects falling under an indefinitely extensible concept $P$, there is a more comprehensive plurality of objects falling under $\mathrm{P}$.

Suppose we translate the quantifiers for any set/for any plurality with a standard quantifier (i.e. a quantifier behaving as in standard classical semantics). According to such a semantics, the domain of such a quantifier is a set. The problem arises because the domain of the quantifier in A must contain any set of objects falling under $\mathrm{P}$. So the domain of the quantifier must be a set containing all sets of objects falling under $P$. But $P$ is IE-1, which means that given any set of objects falling under $P$ we can find a larger set of Ps. Therefore, a set that contains all set of Ps cannot exist. The same applies to the case B.

What about if we were to interpret for any set/for any plurality with a plural quantifier $\forall x x$ ? This requires that the objects over which 'for any' ranges form a plurality. In the case $A$, this works: for any set can range over the plurality of all sets of objects falling under $P$. Here $P$ is $\mathrm{IE}-1$, which means that there is no set of all its instances. But this is compatible with the existence of the plurality of all its instances ${ }^{8}$. By contrast, this approach cannot work with $B$. The problem arises because the domain of the quantifier in B must contain any plurality of objects falling under $\mathrm{P}$. So the domain of the quantifier must be a plurality containing all plurality of objects falling under $\mathrm{P}$. But $\mathrm{P}$ here is IE-2, which means that given any plurality of objects falling under $\mathrm{P}$, we can find a larger plurality of Ps. Therefore, a plurality that contains all sets of Ps cannot exist.

\footnotetext{
7 Of course, I am not presenting such conceptions of indefinite extensibility as interpretations of what Dummett had in mind. I am not interested here in Dummett's exegesis, so I shall not concern myself with the question of whether the conception of indefinite extensibility presented here faithfully represents his views.

8 This was George Boolos's position concerning the concept of set: there is no universal set, but there is the plurality of all sets.
} 
The fact that the plural interpretation of the quantifiers is compatible with IE- 1 shows that IE-1 still represents an extensional way of interpreting indefinite extensibility (keep in mind that here we are using the notion of plurality as an extensional notion). The concept of set may be IE-1, since given a set we are always able to specify a more comprehensive one, but we may consider all sets as forming a plurality. In other words, the concept of object will turn out not to be indefinitely extensible: after all, in this context, it has a determined extension - the plurality of all objects.

$\mathrm{IE}-1$ is not the notion of indefinite extensibility that we are interested in here. Our concern in the present work is IE-2. The reason is that our solution to the paradox of nothingness claims that the same concept of object is indefinitely extensible, i.e. there is no all-inclusive plurality of objects. As soon as we tried to specify (quantify over) the plurality of all objects, we are able to specify new objects, not belonging to that plurality. As we shall see in $\S 4$, the notion of nothingness provides a way of expanding any plurality of objects. From now on, I will always take domains for quantifiers to be pluralities of objects.

\subsection{Indefinite extensibility and generality}

The idea of IE has been developed in different ways in the literature, with different proposals on how to interpret generality over it. For instance, according to Dummett, indefinite extensibility must be interpreted in a constructivist way, and generality over an indefinitely extensible sequence requires an interpretation of quantifiers as in intuitionistic logic. However, since our main target here is the problem of nothingness, we should find a reading of IE suitable for dealing with such a problem. As I shall explain later, the problem of nothingness is a problem of individuation of an object (the object denoted by the noun phrase 'nothingness') that arises as soon as we refer to everything. We thus need an interpretation of IE in terms of individuation of further and further objects. This is the key idea behind Linnebo's approach to indefinite extensibility (Linnebo 2010, 2013, 2018). Linnebo interprets an indefinitely extensible sequence exactly in terms of the individuation of its elements. Suppose you fix a (plural first-order) language, with some objects as a universe of discourse. Then you can quantify over these objects with plural quantifiers. If in your language there is an indefinitely extensible concept, then by referring to all its instances (in the domain of the language) you will be able to individuate a further object, not in the original domain, which nevertheless instantiates that concept (recall that an indefinitely extensible concept is associated with a principle of extension). In other words, you will have all the linguistic and semantic resources to refer to/individuate an object not in the original 
universe of discourse. It is important to notice here that as soon as you refer to this new object, you are already 'inside' a more comprehensive domain of discourse, i.e. the individuation of this object immediately expands the original plurality in a more comprehensive one. The consequence of such an interpretation is that indefinite extensibility turns out to be compatible with the Law of Excluded Middle. In fact, to say that the law fails for a certain object $a$ presupposes that the object $a$ has already been individuated. But this means that the object $a$ is a member of the current universe of discourse, and so either it falls under the indefinitely extensible concept, or it does not, which contradicts the fact that the law of excluded middle fails for it. We are thus not forced to accept intuitionist logic when dealing with the interpretation of IE in terms of individuations of further and further objects.

Since each plurality can be enlarged according to IE-2, we need a way to generalize over any plurality. We already know that standard and plural quantification will not do. However, the principle of extension gives us a general procedure that, if applied to a certain plurality, yields a more comprehensive plurality. It is this principle that assures us the possibility of stating general truths that do not depend on the objects actually present in our domain of discourse, but which holds no matter the plurality we are considering. In this way, this is a purely intensional form of generality. To formally capture this form of generality, Linnebo introduces a primitive modal operator that must be combined with the quantifiers. The 'modalized quantifier' $\square \forall$ indicates that, no matter what domain you are considering, the sentence in the scope of the modality will turn out to be true. For example, if you defend the idea that any plurality can form a set, then no matter what plurality you are considering, you can always individuate a further object, not belonging to that plurality, i.e. the set whose elements are all and only the members of the considered plurality ${ }^{9}$ (conversely, the modalized quantifier $\diamond \exists$ expresses the idea of potential existence: it is possible to expand the domain in such a way that the expanded domain contains an object of a certain kind). The modal operators that are part of the modal quantifiers do not express the standard notions of metaphysical or logical necessity/possibility. We are dealing here with a different kind of modality: the possibility of expanding the domain in a certain way or the fact that a certain result will occur however the domain is expanded ${ }^{10}$.

\footnotetext{
9 This principle is known as modal collapse and has been defended by Linnebo 2010.

10 More on this can be found in Linnebo 2013, 2018; see also Fine 2006. I explained my preferred way of interpreting such a form of generality in [...quotation delated for peer-review], to which I refer the reader for a
} 
It is important to note that a sentence with the modalized quantifier $\square \forall$ is always valuated with regard to a certain plurality of objects that functions as a domain of discourse. As in the standard Krikpean semantics, a modal sentence is evaluated with regard to a domain of objects (a possible world) and to the accessibility relation, here sentences with modalized quantifiers are evaluated with regard to a specific domain of objects (and to the accessibility relation as well). However, if the sentence is true, its truth will be independent from the objects present in that domain (and from the objects of the accessible domains) and in virtue of this the sentence manages to generalize over any domain. But, and this is a crucial point to understand, such generalization over objects not yet individuated does not make available (i.e. does not provide the individuation of) these objects. If I claim $\square \forall x(x=x)$ and this is a true claim, I am claiming something true for all objects individuated, but also for those not individuated yet. But this does not provide an individuation of these objects. Compare: in intuitionistic logic, I can assert a universal sentence like 'every ordinal has a successor' if I have a procedure such that given an ordinal, it gives me its own successor. But this does not imply that I have actually constructed all the ordinals.

\subsection{Collective versus distributive generality}

The generality expressed by the modalized quantifier is a form of absolute generality. However, it differs from standard quantification since it does not require an absolute domain (set or plurality) of quantification. One of the consequences of such difference is that generality over an indefinitely extensible sequence can only be understood in distributive terms, and not in collective terms. The distinction is well-known: consider the sentence 'The boys are three' (i.e. 'there are three boys'). The predicate 'being three' is a collective predicate: it is predicated to all the boys simultaneously, and not to each single boy. Each single boy is one, not three. On the contrary, if I say 'the boys are 1.75 meters high' I will probably intend that each single boy is 1.75 meters, and not that all the boys taken together (i.e. the sum of their heights) are 1.75 meters high. In this latter case, the predicate is distributive.

It must be noticed that this distinction is somehow orthogonal to the possibility of collecting together in a domain all objects of a certain type. With this I mean that the two sentences can be both formally translated by means of quantifiers that range over fixed domains. The 
first sentence will become $\exists x \exists y \exists z$ ( $x$ is a boy \& $y$ is a boy \& $z$ is a boy \& $x \neq y \& x \neq z \& y \neq$ $z$ ), where the domain of the quantifiers is the set composed of the three boys. The second sentence can be translated as follows: $\forall x$ ( $x$ is a boy $\rightarrow x$ is 1.75 meters high). The domain of the quantifier can be whatever set I am referring to. Therefore, both collective and distributive predications are compatible with the possibility of collecting together in a fixed domain the objects of which we are speaking. This is nothing surprising.

It is more important to observe that if it is not possible to collect together all elements of a certain kind into a single domain, then we cannot refer to them by means of collective predicates. The reason for this is straightforward: a collective predicate applies to a collection of individuals, but if there is no collection, then a fortiori the predicate cannot apply. What remains are distributive predicates, which do not require that the objects to which they apply form a collection. In this sense, generalization over an indefinitely extensible sequence must be understood in distributive terms (and so the modalized quantifiers must be understood distributively). The modalized quantifiers do not allow us to generalize over the collection of all elements of an indefinitely extensible concept (since such a collection does not exist), but rather they allow us to generalize distributively over each element that falls under such a concept. Therefore, I can say something of each element of an indefinitely extensible sequence (and in this way I manage to speak of the whole sequence, because what I am saying - if true - is true for each single member of the sequence, even those elements that have not been constructed or individuated yet), even if such elements cannot be all collected together into a domain, since I will always be able to individuate further and further objects.

\section{§3. Nothingness and the contradiction}

It is now time to introduce the notion of nothingness and to show how it naturally leads to a contradiction. In doing so, I will rely on Graham Priest's account of nothingness (as exposed in Priest 2014, 2018). However, I shall introduce some simplifications, since it is not this account that I intend to defend (on the contrary, my solution seeks to be a direct rival of Priest's own account). In particular, I shall not endorse Priest's proposal of interpreting nothingness as the empty fusion, i.e. the (mereological) fusion of the elements of the empty set. The reason will become clear when I explain my own solution. Moreover, no reliance on Priest's noneism will be made, i.e. his own version of neo-Meinongianism, which shows that my own account does not rely on a neo-Meinongian ontology. While Priest 
explicitly develops his own account within a neo-Meinongian framework, an account of nothingness can also be developed within a standard (Quinean) meta-ontology. As a consequence, the three premises below can be accepted independently on one's favorite meta-ontology. Of course, the meta-ontology one chooses will influence the interpretation of some key elements of those premises. More specifically, the interpretation of the quantifiers and the notion of object will be affected by the underlying meta-ontological view. It is worth briefly discussing the latter. The argument assumes a very broad concept of object: everything whatsoever is an object. We shall use the predicate $O(x)$ to be read as ' $x$ is an object', and we shall define it in the following way: $O(x)={ }_{\operatorname{def}} \exists y(y=x)$, which simply means that something is an object if there is something to which it is identical. Within a Meinongian perspective, one defends the idea that each mental state is intentional directed towards an object - Jacquette (2015) has called this principle 'the Intentionality Thesis'11. Everything we can speak of is thus an object: we have both existent and non-existent objects (consequently, the quantifiers will be interpreted as existential-neutral). In such a scenario, the referent of the singular term 'nothingness' will be probably interpreted as a non-existent object $^{12}$. Of course, things are quite different within a Quinean meta-ontology, where everything exists. The quantifiers are not ontologically neutral, and the idea of non-existent objects is regarded as simply mistaken. However, it is also true for the Quinean that everything is an object; the concepts 'existing thing' and 'object' coincide. In reading the premises below, one can thus interpret the concept of object and the quantifiers according to one's preferred meta-ontology; in any case, as soon as she acknowledges the legitimacy of treating the term 'nothingness' as a noun phrase, she should deal with the paradox of nothingness.

My account is based on the following three premises.

1. There are occurrences of 'nothingness' as a noun phrase not reducible to quantification; the semantic contribution of a noun in a sentence is the object the noun refers to.

2. When speaking of everything and nothingness, quantifiers must be taken as absolutely unrestricted.

\footnotetext{
11 See also Casati and Fujikawa 2017, §1.

12 Voltolini 2015 explicitly interprets it as a non-existent object. Moreover, he argues that it is an inconsistent object, and so one can accept it only in the case one accepts an ontology of impossibilia.
} 
3. Nothingness is the lack of everything or, which is the same, the absence of everything.

\subsection{Discussion of premise 1}

Premise 1 is the basic premise of any account of nothingness. If nothingness were always reducible to quantificational generality (no things, no objects), then our present problem will simply disappear. Carnap (1932) famously argued that every occurrence of 'nothing' as a noun phrase in a sentence must be translated, in a formal language that wants to capture its logical form, by means of a quantifier (and negation as well). This position has been the orthodoxy in analytic philosophy until recently, which has seen a number of defenses of the idea $^{13}$ that there are occurrences of 'nothing' as authentic noun phrases. Such defenses point to sentences where the term 'nothing' cannot be translated with a quantifier without modifying the intending meaning of the sentence ${ }^{14}$. Since I find such defenses quite strong, and I have nothing to add to them, I shall not discuss this problem here. Instead, I will just assume that this is in fact true and develop my account ${ }^{15}$. From now on, I will use 'nothingness' as a noun phrase.

Recognizing that 'nothingness' sometimes occurs as a noun phrase has important consequences. A (singular) noun phrase is a singular term that denotes an object. The semantic contribution of a singular term to the sentence in which it occurs is the object to which the noun phrase refers. The connection between singular terms and objects is so tight that Frege even characterized objects as possible referents of singular terms (he used to speak of nouns, with which he meant proper nouns and definite descriptions). It is thus natural to suppose that if 'nothingness' has proper occurrences as a noun phrase, then these occurrences refer to/denote an object.

\footnotetext{
13 For example, Voltolini 2015, Priest 2014, 2018, Oliver and Smiley 2013a, and Simionato 2017.

14 Perhaps the most famous of Priest's examples is the following: consider the sentence 'God created the universe out of nothing', and translate it by means of a quantifier: there is nothing from which God created the universe. But this is true also in the case in which the universe is eternal and God never created it. Therefore, the quantificational translation gives us a different sentence that can have a different truth-value with regard to the original one. This should show that in the original sentence 'nothing' is not quantifier, but a true noun phrase. However, this particular example has been challenged by Sgaravatti and Spolaore 2018, who provide an interpretation of the sentence without assuming 'nothing' as a singular term. As such, the example cannot be considered conclusive.

${ }_{15}$ Consider the following pun: 'Nothing is bigger than the universe; my hand is bigger than nothing, so my hand is bigger than the universe'. The pun is built on the different logical functions of the term nothing, respectively as a quantifier phrase and as a noun phrase. Of course, the derivation (my hand is bigger than the universe) is fallacious, precisely because of this equivocation, but the fact that the pun works is a clear clue that both uses of 'nothing' (as a quantifier and a noun phrase) are legitimate, at least in natural language.
} 
However natural this suggestion might be, one could resist such a conclusion by pointing out that there could be non-denoting singular terms. As a matter of fact, Frege's characterization of an object as a possible referent of a singular term does not imply that every singular term denotes. This is the path taken by Oliver and Smiley (2013a). They argue in favor of the legitimacy of the use of 'nothingness' - or 'Zilch' as they call it - as a singular term, and of the impossibility of reducing all occurrences of nothingness to quantification. But they deny that the occurrences of 'nothingness' as a noun phrase have a referent. In particular, according to them, 'nothingness' does not refer to a non-existent object or to an impossible object. It is simply an empty term. As a matter of fact, if the term 'nothingness' referred to an object, it would refer to something, which seems contradictory. This is in fact another way of expressing the paradox of nothingness, and Oliver and Smiley's proposal to accept empty terms is a way of resolving the paradox. Though this suggestion may seem natural, there are reasons to resist it. In particular, a Meinongian is committed to the Intentionality Thesis which implies that if we speak or think of anything, then we are referring to an object. But we speak of nothingness, and so the term must refer to an object of some kind. The term 'nothingness' for her is a referring term. Such an easy reply is not available for the Quinean. It is true that empty terms have not enjoyed much favor in the history of analytic philosophy ${ }^{16}$, and the Russell-Quine strategy of paraphrasing negative existential sentences seems to presuppose that no truly singular term is empty. But these are not arguments against empty terms. A Quinean could note that the allowance of empty terms (empty nouns) implies that the semantic contributions of nouns are not more than (or not only) the objects to which the nouns refer. But if this is the case, it is not at all clear what this semantic contribution consists in. A homogeneous treatment of singular terms is thus preferable, in particular if it can be shown that the paradox of nothingness can be consistently solved. Once a consistent solution has been exhibited, the Quinean can claim that he was not forced to abandon a homogeneous treatment of singular terms just to avoid the paradox of nothingness.

\subsection{Discussion of premise 2}

Premise 2 expresses another basic assumption of any account of nothingness, i.e. that we truly manage to refer to absolutely everything. Clearly this is the premise I intend to

${ }^{16}$ Russell even defined as proper names the demonstrative exactly because they always have a referent. 
challenge as my previous discussion of indefinite extensibility shows. However, as should be clear, I do not intend to challenge the possibility of absolutely general discourses, but simply the standard way in which they are thought of.

\section{$\underline{3.3 \text { Discussion of premise } 3}$}

Premise 3 is the definition of nothingness that we are working with. The term 'nothingness' denotes the absence of everything. This seems a very natural definition; basically, one may think of nothingness as what remains when we subtract away from reality every object that composes $i^{17}$. The quantifier that appears in this definition must, of course, be totally unrestricted, if we want to be sure that after this 'subtraction', what remains is really nothing.

\subsection{From nothingness to contradiction}

It is useful to allow ourselves some basic mereological notions to reason on everything and nothing. Let us indicate the strict part-of relation by means of the symbol ' $<$ '. We have:

$$
\begin{aligned}
& \left.x<y \text { (to read ' } x \text { is a proper part of } y^{\prime}\right) \\
& x \leq y={ }_{\text {def }}(x=y) \text { or } x<y
\end{aligned}
$$

Then we may define the notion of overlapping as follows:

$$
x \circ y={ }_{\text {def }} \exists \mathrm{z}(\mathrm{z} \leq x \& z \leq y)^{18}
$$

Premise 3 states the definition of nothingness as the absence of everything. If we indicate with $\boldsymbol{n}$ the object denoted by the noun phrase 'nothingness' (and that can be formally defined as $\iota y . \forall x \sim(x \circ y))^{19}$, then from the definition we have that

$\boldsymbol{x} \circ \boldsymbol{n}=\exists \mathrm{y}(\sim \mathrm{Oy} \& \mathrm{x} \circ \mathrm{y})$, where $O y$ means ' $y$ is an object'. This means that no object overlaps $\boldsymbol{n}$ (which is a clear consequence of our definition of nothingness and of the fact that everything is an object).

\footnotetext{
17 I have in mind something like the subtraction argument formulated, for instance, by Baldwin 1996. However, Baldwin's formulation presupposed that reality is composed of a finite number of objects in order to perform the subtraction. I will relax such a condition, and I will assume that, ideally, the subtraction can happen even if the number of objects that composes reality is infinite. In any case, nothing of what I say strongly depends on such an argument; the subtraction argument can just be used to have an intuitive idea of what 'nothingness' should denote.

18 Of course, whether or not the quantifiers used in these definitions are ontologically neutral will depend on the meta-ontology one prefers.

19 That there is such an object is guaranteed by premise 1 .
} 


$$
\begin{aligned}
& \boldsymbol{n} \circ \boldsymbol{n}=\exists \mathrm{z}(\mathrm{z} \leq \boldsymbol{n} \& \mathrm{z} \leq \boldsymbol{n}) \\
& \boldsymbol{n} \leq \boldsymbol{n}=((\boldsymbol{n}=\boldsymbol{n}) \text { or }(\boldsymbol{n}<\boldsymbol{n})) .
\end{aligned}
$$

With this machinery in play, Priest $(2014,2018)$ proves that $\boldsymbol{n}$ is a contradictory object, i.e. he proves that $\boldsymbol{n}$ is an object and is not an object.

1) $\boldsymbol{n}$ is an object.

Everything is an object: $\forall x O x$, from which follows (by universal instantiation) that $O \boldsymbol{n}$.

\begin{tabular}{|c|c|c|c|}
\hline 1 & (1) & $\sim \exists x \sim O x$ & Since everything is an object. \\
\hline 2 & (1) & $\forall x \sim x \circ \boldsymbol{n}$ & From 1, by definition of $\boldsymbol{n}$ \\
\hline 3 & (1) & $\sim \boldsymbol{n} \circ \boldsymbol{n}$ & From 2, by Universal Instantiation \\
\hline 4 & (1) & $\sim \exists \mathrm{z}(\mathrm{z} \leq \boldsymbol{n})$ & $\begin{array}{l}\text { From 3, by definition of overlapping applied to } \boldsymbol{n} \text { : } \\
\boldsymbol{n} \circ \boldsymbol{n}=\exists \mathrm{z}(\mathrm{z} \leq \boldsymbol{n} \& \mathrm{z} \leq \boldsymbol{n})\end{array}$ \\
\hline 5 & (1) & $\sim \boldsymbol{n} \leq \boldsymbol{n}$ & From 4, by definition of overlapping applied to $\boldsymbol{n}$ \\
\hline 6 & (1) & $\boldsymbol{n} \neq \boldsymbol{n}$. & From 5 , for the definition of $\leq$ \\
\hline 7 & (7) & $x=\boldsymbol{n} \vee x \neq \boldsymbol{n}$ & Reasoning by cases \\
\hline 8 & $(1,7)$ & $x \neq \boldsymbol{n}$ & From 6 and 7 (first disjunct) \\
\hline 9 & $(1,7)$ & $x \neq \boldsymbol{n}$ & From 7 (second disjunct) \\
\hline 10 & (1) & $x \neq \boldsymbol{n}$ & From $7,8,9$ by Elimination of $\mathrm{v}$ \\
\hline 11 & (1) & $\forall x x \neq \boldsymbol{n}$ & From 10, by Universal Introduction \\
\hline 12 & (1) & $\sim O n$ & From 11, by definition of 'being an object' \\
\hline
\end{tabular}

2) $\boldsymbol{n}$ is not an object.

The numbers in the first column indicate the line of the derivation, while the second column tracks the logical dependence between the various lines of the derivation. The numbers present in the explanation in the right column refer to those of the first column.

Putting together the two derivations, we both have $O n$ and $\sim O n$. Nothingness is a contradictory object, because it is and is not an object.

\section{§4. Nothingness in an indefinitely extensible world}


What we have done is to derive a contradiction from the premises above. Usually when faced with a contradiction, one is forced to abandon one of the premises. Here I will suggest that there is in fact something to revise in the derivation above, namely the thesis that quantifiers are to be taken as totally unrestricted. My attack on nothingness (and on Priest's account) is composed of two steps. First, I shall show how to restore consistency by challenging premise 2 . The result will be the exploitation of the contradiction to show the existence of a particular indefinitely extensible sequences of objects. This will give us a relative notion of nothingness, in the sense that each plurality of things will be associated with its own nothingness, i.e. with the absence of all things that compose that specific plurality. Second, I shall exploit the formal machinery introduced in $\S 2$ regarding generality over an indefinitely extensible sequence to dissolve the problem of the absolute notion of nothingness. I shall argue that once we take seriously the idea of indefinite extensibility, then there is no room for the problem of nothingness. In other words, the problem arises because we tacitly presuppose that reference to everything requires the existence of an allinclusive plurality.

\subsection{What if premise 2 is false?}

The argument above is to the effect that nothingness both is and is not an object. The quantifiers in the argument range unrestrictedly over all objects, and therefore $\boldsymbol{n}$ turns out to be both inside and outside the range of the quantifiers. There is a totality (the totality of everything) and $\boldsymbol{n}$ is both inside and outside this totality.

It is here that the idea of indefinite extensibility suggests a way through this impasse. What can play the role of the principle of extensibility in the present context? And, more importantly, which concept will turn out to be indefinitely extensible? Since the contradiction shows that $\boldsymbol{n}$ is and is not an object, i.e. it both falls and does not fall under the concept of object, the concept in question can only be the concept of object. Here the idea is to exploit the contradiction derived above as a sort of extensibility principle for the concept of object, and this is possible if we use the derivation of the contradiction as a reductio of premise 2 , i.e. of the idea that the quantifiers were totally unrestricted. The friend of indefinite extensibility may argue as follows: when we developed the argument, we supposed that our quantifiers were totally unrestricted. However, we produced a sound argument to the claim that $\boldsymbol{n}$ is not an object, which means it is not in the range of our quantifiers, since they range over objects. But $\boldsymbol{n}$ must be an object. To preserve consistency, we can abandon the idea 
that our quantifiers were absolutely unrestricted; in this way, the proof that $\boldsymbol{n}$ is not an object becomes the proof that $\boldsymbol{n}$ is not an object in the range of the quantifiers. So we can conclude that $\boldsymbol{n}$ was not in the range of our quantifiers but still it is an object. The contradiction shows us that the object $\boldsymbol{n}$ cannot be in the range of our quantifiers.

To see that this is in fact a possible way out of the paradox, let us go back to Priest's argument above, and suppose that the quantifiers in that argument are restricted to a certain non-inclusive domain $D$. The argument now becomes as follows:

\begin{tabular}{|c|c|c|c|}
\hline 1 & (1) & $\forall y O y$ & Everything is an object \\
\hline 2 & (1) & $\sim \exists x \sim O x($ in $D)$ & Everything is an object (in $D$ ). \\
\hline 3 & (1) & $\forall x \sim x \circ \boldsymbol{n}$. & From 2 , by definition of $\boldsymbol{n}$ ( $x$ ranges over $D)$. \\
\hline 4 & (4) & $\boldsymbol{n} \prec D$ & Assumption ( $\boldsymbol{n}$ is in $D)$ \\
\hline 5 & $(1,4)$ & $\sim \boldsymbol{n} \circ \boldsymbol{n}$ & From 3 and 4 by Univ. Inst. \\
\hline 6 & $(1,4)$ & $\sim \exists \mathrm{z}(\mathrm{z} \leq \boldsymbol{n})$ & From 5, by $\boldsymbol{n} \circ \boldsymbol{n}=\exists \mathrm{z}(\mathrm{z} \leq \boldsymbol{n} \& \mathrm{z} \leq \boldsymbol{n})$ \\
\hline 7 & $(1,4)$ & $\sim \boldsymbol{n} \leq \boldsymbol{n}$ & From 6, by def. of overlapping applied to $\boldsymbol{n}$ \\
\hline 8 & $(1,4)$ & $\boldsymbol{n} \neq \boldsymbol{n}$. & From 7 , for the definition of $\leq$ \\
\hline 9 & (9) & $x=\boldsymbol{n} \vee x \neq \boldsymbol{n}$ & Reasoning by cases \\
\hline 10 & $(1,4,9)$ & $x \neq \boldsymbol{n}$ & From 8 and 9 (first disjunct) \\
\hline 11 & (9) & $x \neq \boldsymbol{n}$ & From 9 (second disjunct) \\
\hline 12 & $(1,4)$ & $x \neq \boldsymbol{n}$ & From $9,10,11$ by Elimination of $v$ \\
\hline 13 & $(1,4)$ & $\forall x x \neq \boldsymbol{n}$ & From 12, by Univ. Intro. (the range of $x$ is $D$ ). \\
\hline 14 & $(1,4)$ & $\sim O n$ & From 13, by definition of 'being an object' \\
\hline 15 & (1) & On & From the fact that everything is an object \\
\hline 16 & (1) & $\sim(\boldsymbol{n}<D)$ & From $4,14,15$ by Intro. \\
\hline
\end{tabular}

The argument is a reductio ad absurdum. The contradiction of lines 14 and 15 is derived from the assumption of line $4(\boldsymbol{n} \prec D)$. Notice that this assumption is essential to apply 
Universal Instantiation to get 5 . In such a scenario, we can easily negate 4 , and conclude that $\boldsymbol{n}$ is an object not in $D$ (line 16).

That $\boldsymbol{n}$ is an object follows from that fact that everything is an object. Now, consider the sentence $\forall x O x$. If the quantifier ranges over $D$, then we cannot conclude $O n$ since the other side of the proof has established that $\boldsymbol{n}$ is not in the range of $D$; if $\boldsymbol{n}$ is in the domain of the quantifier (for instance, if the domain is $D^{\prime}=D \cup\{\boldsymbol{n}\}$ ) then we can conclude that $O \boldsymbol{n}$. But if we now rerun the other side of the argument with regard to this new domain $D^{\prime}$, we are able to find a new object $\boldsymbol{n}^{\prime}$ (with $\boldsymbol{n}^{\prime} \neq \boldsymbol{n}$ ) that is not in the domain $D \cup\{\boldsymbol{n}\}$.

\subsection{More than one nothingness?}

It is important to understand that if we run the proof with regard to different domains, we are able to define different objects that play the role of nothingness ${ }^{20}$. This may seem puzzling at first, but I think that the strangeness of such a claim will immediately disappear as soon as we reflect on the notion of nothingness we are working with. We defined nothingness as the lack or absence of everything. Therefore, the quantifier 'everything' is essential to the same notion of nothingness. Now, if the domain of that quantifier is restricted, then the notion of nothingness will be a relative notion, i.e. it would be relative to the particular domain we take its quantifier to range over. By changing this domain, we will change also the notion of nothingness. Let us consider two different domains, for example $D$ and $D \cup\{\boldsymbol{n}\}$, and let us consider the two respective definitions of nothingness:

$$
\begin{aligned}
& \boldsymbol{n}=\text { the absence of every element in } D \\
& \boldsymbol{n}^{\prime}=\text { the absence of every element in } D \cup\{\boldsymbol{n}\}
\end{aligned}
$$

These two objects are clearly different objects. Metaphorically, if we think of $\boldsymbol{n}$ as the result of subtracting all elements of $D, \boldsymbol{n}^{\prime}$ will be the result of subtracting all elements of $D \cup$ $\{\boldsymbol{n}\}$, and thus we must subtract the same $\boldsymbol{n}$ to obtain $\boldsymbol{n}^{\prime}$. If consistency must be preserved, the $\boldsymbol{n}$ and $\boldsymbol{n}^{\prime}$ must be different.

\footnotetext{
20 This is clearly the reason why we dropped Priest's characterization of nothingness as the mereological fusion of the empty set. Since there is only one empty set, then there will be only one empty fusion, and so only one nothingness. On this point one might complain that we are actually cheating. Since our characterization of nothingness differs from Priest's, it is not a solution to a paradox to show that the paradoxical argument fails for something else. However, as can easily be appreciated by looking at the derivation of the paradox above, Priest's identification of nothingness with the fusion of the empty set does not play any role in that derivation. As such, our proposal is a direct solution to the paradox above, which is essentially the same paradox considered by Priest. Moreover, if one wants to block our solution by assuming that nothingness is the fusion of the empty set, one should argue for such a claim, otherwise the assumption would beg the question against the present proposal.
} 
We can easily state a criterion of identity for such objects. Let us consider $\boldsymbol{n}$ and $\boldsymbol{n}^{\prime}$ which are respectively the absence of everything in $D$ (shorter: $a(D)$ ) and the absence of everything in $D^{\prime}$ (shorter: $a\left(D^{\prime}\right)$ ). So $\boldsymbol{n}=a(D), \boldsymbol{n}^{\prime}=a\left(D^{\prime}\right)$. Then we can state the following criterion of identity:

$$
\boldsymbol{n}=\boldsymbol{n}^{\prime} \leftrightarrow \forall x\left(x<D \leftrightarrow x<D^{\prime}\right)
$$

which by Ext-P means that $\boldsymbol{n}=\boldsymbol{n}^{\prime}$, when $D \equiv D^{\prime}$. Notice that $a(\ldots)$ is an operator that applies to domains of objects and yields singular terms referring to the nothingness of that specific domain.

At this point we are in a position to appreciate our account of nothingness and why it gives rise to an indefinitely extensible sequence. Let our language $L$ be the language of an ordinary plural logic like PFO with identity $(=) . \mathrm{L}$ is associated with a domain of objects $D$, which includes, by supposition, all the objects there are. Clearly in this language we have all the resources that we introduced above to speak of nothingness, such as the congruence relation (三) and the mereological notions. We then have an operator $a(x)$ to be read as 'the absence of everything in the domain $x^{\prime}$ that applies to pluralities of objects (domains) to yield singular terms $a(D), a\left(D^{\prime}\right), a\left(D^{\prime \prime}\right) \ldots$ denoting the objects $\boldsymbol{n}, \boldsymbol{n}^{\prime}, \boldsymbol{n}^{\prime \prime}, \ldots$ which are respectively the absence of everything in $D, D^{\prime}, D^{\prime \prime} \ldots$ For instance, if $a(x)$ is applied to the objects $b b-$ $a(b b)-$, it yields an object which is the absence of all the $b b$. Since the domain $D$ has been taken as all-inclusive, these objects are members of the domain. And in fact, when the operator $a(x)$ has been applied to a less than all-inclusive plurality $D^{x}$, there is no inconsistency in claiming that the resulted object $\boldsymbol{n}^{\boldsymbol{x}}$ is in the domain of L. But the plural quantifier of $L$ allows us to generalize over all elements of $D$. As such we can apply the operator to $D$, i.e. to all the objects there are. We obtain an object $\boldsymbol{n}$ which is the absence of all elements of our domain. And here we face the contradiction that we derived in $\S 3.4: \boldsymbol{n}$ is and is not an object. Consistency is restored by claiming that $\boldsymbol{n}$ is for sure an object, but not one of those in the original plurality: $\sim(\boldsymbol{n} \prec D)$. This means that the individuation of $\boldsymbol{n}$ has the effect of expanding the original domain into a more comprehensive one.

There are some things to notice here. First, the derivation of the contradiction is essential to claim that $\sim(\boldsymbol{n} \prec D)$, which means that such a derivation is essential to the individuation of $\boldsymbol{n}$. In other words, $\boldsymbol{n}$ is individuated as the object that makes the domain expand, and this is possible precisely because $\boldsymbol{n}$ cannot be in the original domain on pain of contradiction. Second, the process can be iterated indefinitely: once $\boldsymbol{n}$ has been added to $D$, we obtain 
the new expanded domain $D \cup\{\boldsymbol{n}\}$ to which we can apply a further operator to obtain the absence of everything in $D \cup\{\boldsymbol{n}\}$. And so on without end. What we obtain is an indefinitely extensible sequence of objects. These objects are relative nothingness (they are the absence of everything in their respective domains), i.e. they are the nothingness relative to the domain in relation to which they have been defined. In this way, we manage to extend everything by literally adding its own nothingness: we are extending everything with nothing(ness)!

It should be clear that the process of expansion is generated by the presence of the notion of nothingness, defined as the absence of everything. In particular, as the formula ıy. $\forall x \sim(x \circ y)$ makes clear, the expansion is a product of two factors: the unrestricted universal quantifiers that range over all elements of the domain, and the presence of negation, which prevents the nothingness from being one of the elements over which the quantifier ranges (as a matter of fact, the notion plays a crucial role in the derivation of the contradiction above). Moreover, this shows how natural our proposal to avoid the contradiction of nothingness is: the claim that $\boldsymbol{n}$ is for sure an object, but not one of those in the original plurality is directly suggested by the characterization of nothingness as an object that is different from all the objects there are: $\iota y . \forall x \sim(x \circ y)$. If consistency must be preserved, nothingness cannot be one of the objects over which $\forall x$ ranges.

\section{$\underline{4.3 \text { An abstractionist account of nothingness }}$}

In the last paragraph, we saw how the expansion works. Given a certain plurality, we can individuate its own nothingness, which turns out to be a new and different object with regard to that plurality. But such an expansion can also be described in slightly different terms. In this section we present this other description, which is in any case equivalent to the one just seen. However, this new description is particularly interesting because it shows that the present account is actually an abstractionist account of nothingness. Therefore, I shall present such a description by means of a comparison with Linnebo's dynamic abstractionist approach to mathematical objects ${ }^{21}$. Let us consider a plural version of the famous Basic Law V:

\footnotetext{
21 Abstractionist approaches are well-known from the literature in philosophy of mathematics. They go back to Frege's attempt to ground arithmetic on second-order logic and an impredicative version of Basic Law V. Principles like Basic Law V are said 'abstraction principles' and they have the form of an equivalence between an identity statement and an equivalence relation over a certain domain. Schematically, $\# \alpha=\# \beta \leftrightarrow \alpha \approx \beta$.
} 


$$
\{x x\}=\{y y\} \leftrightarrow \forall u(u \prec x x \leftrightarrow u \prec y y)
$$

by Ext-P (see §2) (V) is equivalent to $\{x x\}=\{y y\} \leftrightarrow x x \equiv y y$. The law claims that the $x x$ are the same as the $y y$ if and only if the set of the $x x$ is equal to the set of the $y y$. Within Linnebo's account, the principle must be predicative, in the precise sense that the objects in the left-hand side of the bi-conditional (the sets of $x x$ and $y y$ ) are not in the range of any quantifier of the right-hand side, i.e. $\{x x\}$ and $\{y y\}$ are not between the objects that form the $x x$ and the $y y$ (Linnebo 2018, p. 97).

The process of expansion is described in two steps (Linnebo 2018, p. 58). Let us start with a plural language (the base language) to which a domain is associated - called the base domain - in which there are the objects on which we abstract. Then we define an extended language obtained by adding to the base language a new sort of variables and terms. The new sort is reserved for reference to the new objects obtained by abstraction on the base domain. These new objects are located in a separate domain over which only variables of the extended sort range. In this first step, this radical separation prevents the new objects from being in the range of the quantifier of the base language, which would yield the paradox.

The second step consists in the merging of the two domains of the first step into a single domain, over which only one sort of variables range. This merging is possible thanks to the extensional nature of pluralities: consider a set $y$ in the extended domain and its elements $x x$. If the base domain contains a set $z$ whose elements are precisely the $x x$ then we identify $y$ and $z$. If not, then $y$ is a new object distinct from every object in the base domain. In this way the merged domain contains all the objects of base domain plus the new objects obtained by abstraction. We can then consider this merged domain as a new base domain and reiterate abstraction to further expand the domain.

In this setting, the crucial role is played by the abstraction operator $\{\cdot\}$. The operator only applies to pluralities of objects in the base domain and yields objects (sets) that are in the extended domain. The functioning of such an operator is regulated by the congruence relation (which is an equivalent relation) of the right-hand side of $\mathrm{V}$. This equivalence relation

\footnotetext{
For a general introduction to the abstractionist's approach, I suggest Cook 2009. Linnebo 2018 provides an account of what he calls 'dynamic abstraction', i.e. the idea that the passage from the equivalence relation to the identity statement in an abstraction principle (i.e. the passage from right to left) may lead to an expansion of the domain over which the equivalence relation has been defined.
} 
is defined for the objects of the base domain: every time we have a congruence relation between pluralities of objects in the base domain, we can apply the abstraction operator which individuates new objects - their correspondent sets. In this scenario, it is the abstraction principle $\mathrm{V}$ that triggers the expansion. Notice that the direction from left to right of $\mathrm{V}$ gives us the identity conditions for the objects that have been introduced (the sets), while the direction from right to left gives us a condition of existence for those sets $(\{x x\}=$ $\{y y\}$ implies $\exists z z=\{x x\})$. Of course, this expansionist account of $V$ only gets off the ground if one accepts a certain conception of set such that a set is determined by its elements, i.e. when some elements are available, we do not need any further resource to introduce the set that exactly comprises those elements as members. In other words, (V) can be seen as an implicit characterization of such a notion of set.

The situation is analogous in our present account. The two-step description of the expansion can just be transported here without any substantial change. In our context, the abstraction is played by the operator $a(x)$. The operator only applies to pluralities of objects in the base domain and yields objects in the extended domain (when the quantifier is taken as totally unrestricted). The functioning of such an operator is regulated by the equivalence relation on the right-hand side of ID-N, which is $\forall x\left(x \prec D \leftrightarrow x \prec D^{\prime}\right)$. Since $D$ and $D^{\prime}$ are pluralities, this exactly expresses the congruence relation between pluralities. This equivalence relation is defined for the objects of the base domain: every time we have a congruence relation between pluralities of objects in the base domain, we can apply the abstraction operator which individuates new objects - their correspondent nothingness. In this scenario, it is the abstraction principle ID-N that triggers the expansion. Notice that the direction from left to right of ID-N gives us the identity conditions for the objects that have been introduced (the nothingness), while the direction from right to left gives us a condition of existence for those objects $\left(\boldsymbol{n}=\boldsymbol{n}^{\prime}\right.$ implies $\left.\exists z Z=\boldsymbol{n}\right)$. As before, this expansionist account of ID-N only gets off the ground if one accepts as legitimate the notion of nothingness as the absence of everything. In other words, ID-N may be seen as an implicit characterization of the notion of nothingness. In a language where such a notion is not present, we cannot introduce it by means of only the right-hand side of ID-N. If we do not admit the legitimacy of $a(D)$ as a singular term denoting $\boldsymbol{n}$, the equivalence relation $\forall x\left(x<D \leftrightarrow x<D^{\prime}\right)$ is not enough to claim that $\boldsymbol{n}=\boldsymbol{n}^{\prime}$. It is only in the presence of the singular term 'nothingness', defined as the absence of everything, that the equivalence relation $\forall x\left(x \prec D \leftrightarrow x<D^{\prime}\right)$ can 
be used to conclude $\boldsymbol{n}=\boldsymbol{n}^{\prime}$. This clearly shows that the expansion is triggered by ID-N, because it is triggered by the notion of nothingness present in it (when $\forall x$ in $\forall x(x<D \leftrightarrow$ $x<D^{\prime}$ ) ranges over all elements of the universe of discourse). This situation should not come as a surprise, for it mirrors the fact that to derive the paradox of nothingness, we need to explicitly allow in our language the term 'nothingness' as a proper singular term.

\subsection{An important remark}

Above we have used the idea of indefinite extensibility to suggest a way of escaping from the contradiction of nothingness. Here it must be made clear that what we have done is not to suppose that the concept of object is indefinitely extensible and, on the basis of this supposition, show that we can accommodate the contradiction that the notion of nothingness implies. Rather, the contradiction has been used to deny one of the premises from which it was derived - that quantifiers were totally unrestricted - which has the effect of making the concept of object indefinitely extensible. In other words, we provided a new argument (different from the standard set-theoretic ones) for the claim that the concept of object is indefinitely extensible.

\section{§5. Dissolving the problem of nothingness}

The result of the preceding analysis is that the concept of object is indefinitely extensible: given a plurality of objects, it is always possible to find a more comprehensive plurality. This is possible because as soon as we refer to (what we supposed to be) everything, we have all the resources to individuate an object - the absence of everything - that cannot be in the range of the quantifier 'everything' on pain of contradiction. But as soon as we have individuated this further object, the original domain has been expanded (by this simple act of individuation). Since this holds for any plurality of objects, nothingness turns out to be a relative notion.

Let us return to the definition of nothingness that we gave above: the lack or absence of everything. Yet, we are arguing that every plurality can be enlarged, i.e. there can be no maximal plurality that can play the role of the domain of the quantifier everything. As such, the definition of nothingness must always be understood with regard to pluralities of objects that can be enlarged. By considering two different pluralities as a domain for the quantifier, we are able to individuate two different objects that play the role of nothingness. 
However, we argued above that once we have embraced the idea of indefinite extensibility, generalization can only be understood in a distributive way. What happens if we interpret everything in the definition of nothingness distributively? Do we manage to reach an absolute notion of nothingness? One might think that we are actually able to reach such a notion, since the problem of nothingness arises when we have absolute generality and negation (captured - in our definition of nothingness - by the notion of absence). But in $\S 2$ we introduced the modalized quantifiers exactly to express absolute generality over an indefinitely extensible sequence. For this purpose, it should be enough to interpret the linguistic expression 'everything' present in the definition of nothingness as a modalized quantifier. In other words, the absolute notion of nothingness would indicate the object denoting the lack of each single object (distributive reading captured by the modalized quantifier) we could ever individuate. In other words, the objection is trying to reproduce the argument that leads to the paradox of nothingness by means of a notion of nothingness defined as follows: $\iota y . \square \forall x \sim x \circ y$. Since absolute general claims over an indefinitely extensible sequence are allowed, these definitions seem perfectly legitimate, which seems to show that our account allows for an absolute notion of nothingness. But this would bring us back to the contradiction.

That this objection fails can be appreciated as soon as we try to express it more formally. If the objection succeeds, we should be able to derive the contradiction affecting the notion of nothingness in the presence of the modalized quantifiers, i.e. the formal tools that we used to express generality over an indefinitely extensible sequence ${ }^{22}$. That contradiction -1 remind the reader - showed that the $\boldsymbol{n}$ is an object and is not an object.

Let us start with the first claim. For sure, we are committed to the idea that, no matter what we are going to individuate, these will be objects: $\square \forall x O x$. Therefore, as soon as we have individuated $\boldsymbol{n}$, we can conclude $O \boldsymbol{n}$ ( $\boldsymbol{n}$ is an object). Of course, before individuating $\boldsymbol{n}$, we cannot conclude that it is an object, for the simple reason that we cannot say anything specific about it.

The problem for the objection concerns the second derivation that should prove that $\boldsymbol{n}$ is not an object. That derivation started as follows: since everything is an object, no thing is not an object: $\sim \exists x \sim O x$. Thus nothing overlaps $\boldsymbol{n}: \forall x \sim x \circ \boldsymbol{n}$. In particular, $\sim \boldsymbol{n} \circ \boldsymbol{n}$. In our modal context, the derivation becomes like that:

22 Of course, ID-N and V should also be understood in modal terms, because they are applicable however you can expand the universe of discourse. 


\begin{tabular}{|cccc|}
\hline 1 & (1) & $\square \forall x O x$ & $\begin{array}{r}\text { Everything, no matter how you expand } \\
\text { the original domain, is an object. }\end{array}$ \\
2 & (1) & $\sim \diamond \exists x \sim O x$ & From 1, by $\forall \equiv \sim \exists \sim$ and $\square \equiv \sim \diamond \sim$ \\
3 & $\mathbf{( 1 )}$ & $\square \forall x \sim x \circ \boldsymbol{n}$ & From 2, by definition of $\boldsymbol{n}$ \\
4 & $\mathbf{( 4 )}$ & $\square \forall x \sim x \circ \boldsymbol{n} \rightarrow \forall x \sim x \circ \boldsymbol{n}$ & Reflexivity axiom ${ }^{23}$ \\
5 & $\mathbf{( 1 , 4 )}$ & $\forall x \sim x \circ \boldsymbol{n}$ & From 3,4 by Elim $\rightarrow$ \\
6 & $\mathbf{( 1 , 4 )}$ & $\sim \boldsymbol{n} \circ \boldsymbol{n}$ & From 5, by Univ. Inst. \\
$\ldots$ & $\ldots$ & $\ldots$ & $\ldots$ \\
\hline
\end{tabular}

Since everything that we can individuate is an object, it is not possible to individuate something which is not an object: $\sim \diamond \exists x \sim O x$. Thus, nothing that we can individuate overlaps $\boldsymbol{n}: \square \forall x \sim x \circ \boldsymbol{n}$. In particular, $\sim \boldsymbol{n} \circ \boldsymbol{n}$. But the passage from $2(\sim \diamond \exists x \sim 0 x)$ to $3(\square \forall x \sim x \circ \boldsymbol{n})$ is fallacious. The passage would have been legitimated only in the case in which $\boldsymbol{n}$ had already been individuated, but in fact $\boldsymbol{n}$ has not been individuated yet. And if $\boldsymbol{n}$ has not been individuated yet, not only is the passage to $\square \forall x \sim x \circ \boldsymbol{n}$ fallacious, but also we cannot instantiate the formula in $5(\forall x \sim x \circ \boldsymbol{n})$ to obtain $6(\sim \boldsymbol{n} \circ \boldsymbol{n})$, which exactly presupposes the availability of $\boldsymbol{n}$.

But why has $\boldsymbol{n}$ not been individuated yet? As we know, in our indefinitely extensible setting, in order to individuate $\boldsymbol{n}$, we must refer to everything and then exploit the contradiction that arises to claim that $\boldsymbol{n}$ is not in the range of everything. The derivation of the contradiction is thus necessary to individuate $\boldsymbol{n}$. But at the step where we go from $\sim \diamond$ $\exists x \sim O x$ to $\square \forall x \sim x \circ \boldsymbol{n}$ the contradiction has not been derived yet, and so $\boldsymbol{n}$ has not been individuated.

Of course, in the standard case of $\S 3$, where quantifiers are taken to range over the plurality of everything, $\boldsymbol{n}$ is available, exactly because quantifiers are taken to range over everything (and the possibility of expanding the domain is not taken into consideration). Therefore, in this standard setting we can derive the contradiction. What this shows is that to derive the contradiction, the idea that quantifiers range over an extensionally definite plurality of objects is essential.

${ }^{23}$ As explained in footnote 11 , the modality is captured by $\$ 4.2$. 
The objection thus fails badly. The general point is that the distributive form of generality combined with indefinite extensibility allows us to say things concerning objects not yet individuated. As explained in $\S 2$, the fact that we can state truths about things that are not individuated yet does not mean that these truths individuate these objects. This means that these not-yet-individuated objects are not available for the quantifier in the characterization of nothingness to range over. Think of the subtraction argument: if the concept of object is indefinitely extensible, then the subtraction procedure can never end, which means that we can never reach the absolute notion of nothingness. What prevents the absolute notion of nothingness is indefinite extensibility, i.e. the fact that the sequence of objects is extensionally undetermined. Since a distributive form of reference is compatible both with collections (pluralities) of objects and an indefinitely extensible sequence, it is not enough to appeal to it in order to obtain the absolute notion of nothingness.

But in this way, the problem of nothingness has disappeared: once it is recognized that the concept of object is indefinitely extensible, there is no room for a contradictory absolute notion of nothingness.

\section{§6. Two objections and two replies}

It is time to deal with two further objections that may be raised with regard to the present account of nothingness ${ }^{24}$. Here is the first objection.

According to the present account, the two sentences

1) There is nothing

2) There is the absence of everything

are equivalent (of course both the quantifier 'everything' in 1 and the term 'nothing' in 2 are relative to some restricted domain $D$ ). The equivalence simply follows from the fact that we have defined nothingness as the lack or the absence of everything (see §3 above). While the direction from 1 to 2 can be interpreted as a 'something from nothing'-transformation which commits us to a relative nothingness ${ }^{25}$, more problematic is the other direction - from 2 to 1 - that takes us from a committal to a non-committal sentence with regard to nothingness. But what forces one to adopt this reading, in particular if one appeals to the

\footnotetext{
24 I have to thank the first reviewer for the first objection, and the second reviewer for the second objection. 25 For the 'something from nothing'-transformation see Shiffer 1996.
} 
idea of restricted quantification? If I open the fridge and I find it empty, I may clumsily express myself by truly saying "There is the absence of everything (in the fridge)", but it would be more natural to simply and non-committally truly say "There is nothing (in the fridge)". The objection points to the fact that it is difficult to justify the equivalence of sentences 1 and 2 given that they have different commitments. While we could make sense of the direction from 1 to 2 , it is far more difficult to make sense of the direction from 2 to 1 , that is of the direction from a sentence that commits us to a relative nothingness to a sentence without such a commitment.

I think that this objection relies on a misunderstanding. The supposed equivalence between 1 and 2 relies on having defined nothingness as the absence of everything. But such a definition applies to the occurrences of nothingness as a singular term, and not to its occurrences as a quantifier. In other words, only if one accepts the legitimacy of nothingness as a singular term can one then define it as the absence of everything. Consequently, we have only two cases. Either 1 and 2 are equivalent, but this means that 'nothing' in 1 is a singular term, and thus both 1 and 2 are committal to a relative notion of nothingness. Or 1 and 2 are not equivalent, because 'nothing' in 1 is a quantifier, and thus 1 and 2 have different committal imports. In neither case do we have an equivalence between sentences with different committal imports.

However, if one admits both the legitimacy of the sentence "There is nothing (in the fridge)" - with 'nothing' used as a quantifier expression - and the sentence "There is the absence of everything (in the fridge)", where 'the absence of everything' is a singular term, then one should allow at least for the theoretical possibility of having a 'something from nothing'-transformation consisting in the bi-conditional equivalence of the kind "There is nothing (in the fridge) if, and only if, there is the absence of everything (in the fridge)", just as one usually allows for a similar 'something from nothing'-transformation consisting in the following bi-conditional equivalence: " $A$ differs from $B$ if, and only if, there is the difference between $A$ and $B$ ", with the problem of ontological commitment that follows from which reading direction of the bi-conditional one chooses. Moreover, one may wonder why - in the case in which one admits that, in this example, the quantifier reading must be preferred the 'something from nothing'-transformation consisting in the bi-conditional equivalence "There is no thing if, and only if, there is the absence of everything" may not occur, with the above problem of ontological commitment arising again. 
Of course, I am happy to admit that in this example the sentence with the quantifier reading is legitimate, and so in this case we have the bi-conditional equivalence "There is nothing (in the fridge) if, and only if, there is the absence of everything (in the fridge)". And the problem of the ontological commitment depending on which direction we read the conditional arises. In general, the problem arises whenever we have a material equivalence between sentences with 'nothing' as a quantifier and 'nothing' as a singular term; however, as argued above, it seems that there are cases where 'nothingness' cannot be paraphrased away in quantificational terms without changing the meaning of the sentence (and possibly its truth-value: an example is discussed in $§ 7)$. In such cases there cannot obviously be any material equivalence between the sentences with 'nothing' as a quantifier and those with 'nothing' as a singular term. It is the existence of such cases that forces us to give an account of nothingness as a singular term; otherwise if every occurrence of 'nothingness' had been paraphrased away in quantificational term, then no account of nothingness would have been necessary, and one could have done just with the quantifier reading. The present account has been developed to explain how the singular and irreducible singular term 'nothingness' works. Therefore, it is exactly for those cases that the account is required and to which it should be applied. For all other cases, i.e. the cases where we are in the presence of a material equivalence, we can just stick to the quantifier reading, which is usually more natural and elegant (as in the current example).

Let us now deal with the second objection, which may be stated as follows. Nothingness, $\boldsymbol{n}$, has been defined as $\iota y . \forall x \sim x \circ y$. When evaluating the referent of this expression with respect to a domain, $D$, the variables range over $D$. This includes the variable $y$. So the term must pick out a member of $D$, contrary to the claim that it doesn't. To see why this is the case, note that line 2 of the argument at $§ 3.4$ has the form $\forall x \sim x \circ \boldsymbol{n}$, saying that this follows from the definition of $\boldsymbol{n}$. Since it is a description, what it follows from is actually a principle concerning definitions: $\exists ! y A(y) \rightarrow A(\iota y(A y))$. The antecedent of the conditional makes clear that the variable $y$ is in the domain of quantification.

I think that the mistake with this objection is to think that the quantifier $\exists$ ! has the same domain of quantification as the quantifier $\forall x$ in $\iota y . \forall x \sim x \circ y$. The argument at $\S 3.4$ is an adaptation of Priest's argument, and in Priest's case the two quantifiers really have the same domain of quantification. But the inconsistency that arises is a good reason to think that the two quantifiers range over different domains. According to our expansionist picture, it is the 
same act of defining the notion of nothingness relative to a domain - say $D$ - that makes $D$ expand (§4.2). As soon as we have defined the nothingness of a certain domain, we have already expanded that domain. Therefore, the quantifier $\exists$ ! ranges over the expanded domain $D \cup\{\boldsymbol{n}\}$ (where $\boldsymbol{n}$ is the nothingness relative to $D$ ) and not simply $D$.

\section{§7. Can we leave without nothing?}

Our account of nothingness has dissolved the absolute notion of nothingness in favor of only a relative one. But can we leave without such an absolute notion? An account of nothingness is required for the semantics of sentences where 'nothing' occurs as a noun phrase. Can our account explain the semantics of such sentences? I think that the answer is fully affirmative.

First of all, note that the success of Carnap's view is clearly due to the fact that most (if not almost all) occurrences of the term 'nothing' are quantificational. Nothingness as a noun phrase rarely occurs ${ }^{26}$. Perhaps the most relevant occurrence is when we speculate about the whole universe and the passage from nothingness to the existence of something. In particular, this happens when we speculate about the (alleged) creation of the world by God. So, let us consider the sentence present in footnote 14, where 'nothing' seems to be a noun phrase: 'God created the world out of nothing (= out of the absence of everything)'. This seems to require an absolute notion of nothingness. However, this is not the case. First, notice that it is not true that God created the world out of the absolute nothingness, since at least there was God himself27. This shows that God cannot be in the range of the quantifier everything. The notion is in any case relative, not absolute. But our account delivers us a relative notion in a slightly different sense: nothingness would be relative not because God is not in the range of the quantifier that defines it, but because nothingness itself is not in the range of the quantifier that defines it. So 'God created the world out of nothing' becomes 'God created the world out of the nothingness of the created world'. Here nothingness = the absence of every created entity. The upshot is that while God created the world (i.e. all the created entities), she does not create that particular object which is the absence of every

\footnotetext{
${ }^{26} \mathrm{~A}$ reviewer pointed out that there are more examples of occurrences of nothingness as a noun-phrase of those discussed in the present paper. For instance, 'Sartre and Heidegger both wrote on nothing', and 'Nothing is something that puzzled many philosophers since Parmenides' are both sentences where 'nothing' is a nounphrase.

27 See Aquinas 1962, I, q. 65, a. 3. For Aquinas, the creation is not ex nihilo causae efficientis et finalis (the efficient and final cause is God himself).
} 
created entity. In other words, God created the world, not the absence of the world. Notice that this is perfectly in keeping with the theological view according to which God creates the world not out of an absolute nothingness, but rather out of the nothingness of the creatures (ex nihilo sui et subiecti) $^{28}$.

\section{§8. Conclusion}

In this paper we offered a new solution to the old problem of nothingness. This new solution has been developed in two steps. The first consists in showing how to resolve the contradiction to which the notion of nothingness gives rise, namely by claiming that the contradiction shows the indefinite extensibility of the concept of object. If we think that absolute generality requires the presence of a maximal plurality of everything, then we can exploit the contradiction of nothingness to show that this cannot be the case, since the alleged maximal plurality can be expanded. This approach leads to a dynamic abstractionist account of nothingness. The second step consists in showing that, once the idea of indefinite extensibility has been accepted, we can have absolute generality without the contradiction connected with the absolute notion of nothingness. We can have absolute generality, because a distributive form of generality does not require the collection of all the elements over which we are generalizing, while the contradiction no longer arises because the indeterminacy of an indefinitely extensible sequence does not allow the definition of the absolute notion of nothingness. The idea of indefinite extensibility allows us to have our cake (absolute generality) and to eat it too (avoid committing to a contradictory notion of nothingness) ${ }^{29}$.

\section{References}

Aquinas, T. (1962). Summa Theologiae. Roma: Alba Editiones Paulinae.

Baldwin, T. (1996). "There Might Be Nothing.” Analysis, 56, 231-238.

Carnap, R. (1932). "Überwindung der Metaphysik durch logische Analyse der Sprache." Erkenntnis, 2, 219-241; English translation in A.J. Ayer (Ed.) (1966). Logical Positivism (pp. 60-81). New York: The Free Press.

\footnotetext{
${ }^{28}$ Such a formula means that the creation is from the nothingness of the creature (ex nihilo sui) and the nothingness of creature's matter (et subiecti). Again, see Aquinas 1962, I, q. 65.

29 I would like to thank Matteo Plebani and two anonymous referees for this journal for helpful comments on previous versions of this paper.
} 
Casati, F. and Fujikawa, N. (2015). "Better Than Zilch?" Logic and Logical Philosophy, DOI: 10.12775/LLP.2015.004.

Casati, F. and Fujikawa, N. (2017). "Nothingness, Meinongianism and Inconsistent Mereology." Synthese.

Cook, R.T. (2009). "New Waves on an Old Beach: Fregean Philosophy of Mathematics Today." In New Waves in Philosophy of Mathematics (pp. 13-34). Ed. Ø. Linnebo and O. Bueno. Basingstoke: Palgrave Macmillan.

Cooper, J.M. (Ed.). (1997). Plato: Complete Works. Indianapolis: Hackett.

Coxon, A.H. (2009). The Fragments of Parmenides: A Critical Text with Introduction, Translation, the Ancient Testimonia and a Commentary. Revised and expanded edition with new translations by Richard McKirahan. Las Vegas: Parmenides.

Dummett, M. (1991). Frege: Philosophy of Mathematics. Cambridge, MA: Harvard University Press.

Fine, K. (2006). "Relatively Unrestricted Quantification." In: Absolute Generality (pp. 20-44).

Ed. A. Rayo and G. Uzquiano. Oxford: Oxford University Press.

Hegel, G.W.F. (1969). Science of Logic. Trans. A.V. Miller. London: Allen and Unwin.

Jacquette, D. (2015). Alexius Meinong: The Shepherd of Non-Being. New York: Springer.

Linnebo, Ø. (2010). "Pluralities and Sets." The Journal of Philosophy, 107(3), 144-164.

Linnebo, Ø. (2012). "Reference by Abstraction." Proceedings of the Aristotelian Society, $112,45-71$.

Linnebo Ø. (2013). "The Potential Hierarchy of Sets." Review of Symbolic Logic, 6(2).

Linnebo Ø. (2017). "Plural Logic." Stanford Encyclopedia of Philosophy.

Linnebo Ø. (2018). Thin Objects. Oxford: Oxford University Press.

Oliver, A. and Smiley, T. (2013a). "Zilch." Analysis, 73, 601-613.

Oliver, A. and Smiley, T. (2013b). Plural Logic. Oxford: Oxford University Press.

Priest, G. (2014). "Much Ado About Nothing." Australasian Journal of Logic, 11(2), 146-158.

Priest, G. (2018). "Everything and Nothing." manuscript.

Sgaravatti, D. and Spolaore, G. (2018). "Out of Nothing." Thought, 7: 132-138.

Shiffer, S. (1996) "Language-Created Language-Independent Entities." Philosophical Topics, vol. 24, n 1, Spring 1996.

Simionato, M. (2014). "Everything for Nothing." Organon F: International Journal of Analytic Philosophy, 4, 458-470. 
Simionato, M. (2015). "The Opening of Hegel's Logic and The Empty Possible World." Revista Opinião Filosófica, 6(2).

Simionato, M. (2017). The Vindication of Nothingness. Germany: Editiones Scholasticae.

Voltolini, A. (2015). "Heidegger's Logico-Semantic Strikeback." Organon F 22 (Supplementary Issue) 2015: 19-38. 